\title{
Synergistic Anti-Proliferation and Anti-Angiogenesis Effects of Sevacizumab on Hepatocellular Carcinoma Cells in Combination with Chemotherapy
}

Lin Liu ( $\sim 101012478 @ s e u . e d u . c n$ )

Southeast University Zhongda Hospital https://orcid.org/0000-0001-7073-610X

Shukui Qin

Nanjing Jinling Hospital: East Region Military Command General Hospital

Zhengcao Liu

Southeast University Zhongda Hospital

Yinghui Zheng

Southeast University Zhongda Hospital

Li Han

Southeast University Zhongda Hospital

Minmin Zhang

Southeast University Zhongda Hospital

Nuo Luo

Southeast University Zhongda Hospital

Yawen Gu

Southeast University Zhongda Hospital

Yu Zhang

Southeast University School of Biological Sciences and Medical Engineering

Ning Gu

Southeast University School of Biological Sciences and Medical Engineering

Primary research

Keywords: Hepatocellular carcinoma, Sevacizumab, VEGF/VEGFR signaling pathway, 5-fluorouracil, Oxaliplatin

Posted Date: August 3rd, 2021

DOI: https://doi.org/10.21203/rs.3.rs-704885/v1

License: (a) (i) This work is licensed under a Creative Commons Attribution 4.0 International License. Read Full License 


\section{Abstract \\ Background}

We previously found that (via inhibition of the VEGF/VEGFR signaling pathway) ckgroundSevacizumab (Sev), an anti-VEGF monoclonal antibody, was proven to have a superior inhibitory effect than bevacizumab (Bev) on the growth of hepatocellular carcinoma (HCC) cells. This study aimed to explore the anti-proliferation and anti-angiogenic effects of Sev on HCC cells in combination with oxaliplatin (OXA) or 5-fluorouracil (5-Fu).

\section{Methods}

In vitro $\mathrm{HCC}$ /endothelial cell growth in different concentration drug was analyzed by MTT assay, DAPI and flow cytometry assay. Cell scratch test, transwell assay, tube formation assay, zebrafish assay, and CAM assay were used to investigate anti-angiogenesis effect of drugs. VEGF mRNA relative expression changes of zebrafish embryos were detected by RT-PCR.A fluorescence imaging system was applied to observe the growth of transplantation tumor and blood vessels in HCC mouse xenografts. Tissue H-E staining and TEM detection were used to detect the tumor cell apoptosis. MVD was detected by immunohistochemical analysis of CD31. ELISA and western-blots were used to detect the cell VEGF/VEGFR pathway and its downstream target activity both in vitro and in vivo.

\section{Results}

In vitro results showed that the combination of Sev with OXA/5-Fu can synergistically inhibit the proliferation of HCC and endothelial cells. Compared with the corresponding monotherapy group, combination therapy showed a stronger effect on inducing apoptosis and cell cycle arrest. In vivo findings revealed that Sev in combination with chemotherapy can synergistically inhibit tumor growth by inducing cell apoptosis in nude mice with HCC xenografts. In addition, the wound healing and transwell migration assays demonstrated that Sev can inhibit the migration of endothelial cell lines in combination with chemotherapy. In vitro tube formation test, zebrafish and chicken embryonic-angiogenic assay, immunohistochemistry, and in vivo fluorescence imaging consistently verified that Sev and OXA/5-Fu can synergistically inhibit the growth of blood vessels, and the underlying mechanism may be associated with inhibition of the VEGF/VEGFR signaling pathway.

\section{Conclusions}

The combination of Sev and chemotherapy is associated with the inhibition of HCC growth and tumor angiogenesis, which may provide a significant biological rationale for evaluating the efficacy of Sev and OXA/5-Fu combination therapy on HCC. 


\section{Background}

Hepatocellular carcinoma (HCC) is the second leading cause of cancer mortality worldwide, and accounts for about $90 \%$ of all primary liver cancers. However, there are no appropriate treatment strategies for patients with advanced HCC, or for those who have progressed to an advanced stage after traditional therapies have failed [1]. Hence, novel treatment approaches for HCC are required. The liver's dual blood supply system comprising the hepatic artery and portal vein make the enrichment of blood vessels one of the pathological characteristics of HCC. The hypervascular nature of the liver is also one of the fundamental causes of high recurrence and metastasis of HCC. To our knowledge, HCC cells secrete a large amount of vascular endothelial growth factor (VEGF), and VEGF receptor (VEGFR) is overexpressed in human HCC tissues, which is closely associated with the disease progression and poor prognosis of HCC $[2,3]$. VEGF autocrine by HCC cells can not only recognize its own VEGFR to directly promote tumor cell growth and metastases, but it can also bind to VEGFR on the surface of the endothelial cells and induce angiogenesis through the paracrine signaling pathway. Therefore, VEGF/VEGFR targeted therapy has attracted significant resources to improve the efficacy of HCC treatment [4].

Bevacizumab (Bev) is the first anti-VEGF monoclonal antibody approved for marketing and has been shown to be effective in the treatment of various solid tumors, including HCC. In the past two decades, although different types of anti-angiogenic agents have been approved, including small-molecule tyrosine kinase inhibitors targeting VEGF and/or other angiogenic or oncogenic signaling pathways, Bev remains the most frequently used and the most extensively characterized angiogenesis inhibitor [5-7]. As a single agent, Bev has shown an objective response rate of $13 \%$ in patients with unresectable non-metastatic HCC [8]. However, because the efficacy of single agents still seems restricted, combination therapies involving different groups of substances (especially cytotoxic chemotherapeutics) have been developed [9]. The first approved Bev regimen to treat metastatic colorectal cancer was used in combination with chemotherapy rather than as a single agent [10]. In addition, it was found that combining Bev with standard chemotherapy also provided a novel and effective therapeutic option for HCC patients with a poor prognosis [11]. In a multicenter phase II trial involving patients treated with Bev plus capecitabine, the overall response rate according to Response Evaluation Criteria in Solid Tumors (RECIST) was 9\% and the disease control rate was $52 \%$ [12]. In another phase II trial using oxaliplatin (OXA), Bev combined with gemcitabine-oxaliplatin (GEMOX) [13] or capecitabine-oxaliplatin (XELOX) [14] resulted in improved response rates by up to $20 \%$.

Sevacizumab (Sev) is a humanized rabbit anti-VEGF monoclonal antibody developed by Jiangsu Simcere Pharmaceutical Co., Ltd. (Nanjing, China). Our previous study revealed that (via inhibition of the VEGF/VEGFR signaling pathway) Sev is more efficient than Bev in the inhibition of tumor growth and angiogenesis in mouse HCC xenografts [15].

In the present study, we further explored the effects of Sev in combination with OXA or 5-fluorouracil (5$\mathrm{Fu}$ ) on the anti-proliferation and anti-angiogenesis of $\mathrm{HCC}$ both in vivo and in vitro. In addition, we 
investigated possible mechanisms underlying these effects. Our findings may provide the experimental evidence and theoretical basis for the application of Sev in anti-HCC clinical practice treatment.

\section{Materials And Method}

\section{Cell lines}

Human HCC cell lines (HepG2, SMMC-7721, and Bel7402) and microvascular endothelial cell line (HUVEC, EAhy926, and HMEC-1) were obtained from the Cell Bank of Type Culture Collection of Chinese Academy of Sciences (Shanghai, China). Human umbilical vein endothelial cells (HUVECs) were purchased from Nanjing KeyGen Biotech. Co. Ltd. (Nanjing, China). Another human microvascular endothelial cell line (HMEC-1) was provided by the Key Laboratory of Thrombosis and Hemostasis, Suzhou University (Suzhou, China). All cells were cultured in appropriate conditions according to the prescribed instructions.

\section{In vitro cytotoxicity assay}

Sev was provided by Jiangsu Simcere Pharmaceutical Co., Ltd. (Nanjing, China). For in vitro assay, experimental groups were set up as follows: Bev group $(0.1 \sim 20 \mu \mathrm{g} / \mathrm{mL})$, Sev group $(0.1 \sim 20 \mu \mathrm{g} / \mathrm{mL})$, 5Fu group $(4 \sim 64 \mu \mathrm{M})$, OXA group (1.25 $20 \mu \mathrm{M})$, Bev + 5-Fu group, Bev + OXA group, Sev + 5-Fu group, and Sev + OXA group. All experiments were performed in triplicate for each condition and were repeated at least four times. After cells were treated with different concentrations of drugs for 24,48 , and $72 \mathrm{~h}$, MTT (Sigma-Aldrich, St. Louis, MO, USA) assay was performed as described previously [15]. The absorbance at $590 \mathrm{~nm}$ was recorded to calculate the inhibition rate of cell proliferation as follows: inhibition rate $=[1-(O D a-O D b) /(O D c-O D b)] \times 100 \%$, where ODa represents the optical density (OD) value of the experimental wells, ODb denotes the OD value of the blank wells, and ODc indicates the OD value of the negative control wells.

Data were calculated from four independent experiments with five replicates. The interaction between two drugs was assessed on the basis of the q value calculated by the Jin's modified Bürgi's formula: $q=$

$E_{A+B} /\left(E_{A}+E_{B}-E_{A}{ }^{*} E_{B}\right)$, where $E_{A+B}$ refers to the inhibition rate of cell growth in the combination therapy group, while $E_{A}$ or $E_{B}$ indicates the inhibition rate of cell growth in each monotherapy group. Besides, $q>1$, $q=1$, or $q<1$ suggested that the two drugs have synergistic, additive, or antagonistic effects, respectively. For cell cycle assay, following $48 \mathrm{~h}$ of the indicated treatment, 4', 6-diamidino-2-phenylindole (DAPI) staining and flow cytometry were respectively performed as described previously [15].

\section{VEGF/VEGFR expression}

VEGF/VEGFR protein expressions in cells, tissues, or in cell culture medium were detected by Western blotting or enzyme-linked immunosorbent assay (ELISA) as described previously [15]. For quantitative reverse transcription polymerase chain reaction (RT-qPCR), total RNA was isolated from zebrafish tissues by TRIzol reagent according to the manufacturer's instructions (Ambion Inc., Austin, TX, USA). RNA was reversely transcribed by the PrimeScript RT Reagent kit (Takara Biotechnology Co., Ltd., Dalian, China) 
and the synthesized cDNA was subjected to qPCR using the Golden SYBR Green Mix (2x) kit (Toneker Biotechnology Co., Ltd., Shanghai, China) according to the manufacturer's instructions. Glyceraldehyde 3phosphate dehydrogenase (GAPDH) was used as the internal control. The PCR was associated with a pre-denaturation at $94^{\circ} \mathrm{C}$ for $5 \mathrm{~min}$, followed by 35 cycles of amplification consisting of denaturation at $95^{\circ} \mathrm{C}$ for $30 \mathrm{~s}$, annealing at $55^{\circ} \mathrm{C}$ for $30 \mathrm{~s}$, extension at $72^{\circ} \mathrm{C}$ for $30 \mathrm{~s}$, and ended by a final extension at $72^{\circ} \mathrm{C}$ for $10 \mathrm{~min}$. Primer sequences used for PCR were as follows: VEGF-F, ccaggagtatcccgatgaga; VEGF$\mathrm{R}$, gctttgacttctgcctttgg; GAPDH-F, actccactcatggccgttac; and GAPDH-R, gagctgaggccttctcaatg. The melting curve analysis was carried out by LightCycler software (Roche Diagnostics, Risch-Rotkreuz, Switzerland).

\section{Migration assay of endothelial cells}

To perform scratch assay, cells in the log phase were seeded into 24-well culture plates at an appropriate density to reach confluency of over $90 \%$ on the next day. A straight line was drawn on the surface of the cells with a $10 \mu \mathrm{L}$ tip in each well. After washing with phosphate-buffered saline (PBS), the indicated drug intervention was performed and images were taken under an inverted microscope at $24 \mathrm{~h}$ post-treatment. The scratch width was measured using Image-J software, and the cell migration distance was calculated as follows: cell migration distance $=$ scratch width before treatment $(0 \mathrm{~h})$ - scratch width after treatment (24 h).

For transwell assay, HUVEC, EAhy926, or HMEC-1 cells were seeded into the upper chambers, and HepG2 cells were seeded into the lower chambers containing a polyethylene terephthalate membrane (Corning Inc., Corning, NY, USA), respectively. After $24 \mathrm{~h}$ of treatment, the migrated cells at the bottom of the upper chamber were fixed with $95 \%$ ethanol for $30 \mathrm{~min}$, stained with $0.1 \%$ crystal violet for $10 \mathrm{~min}$, and counted from 6 random fields under an inverted microscope (magnification 200x; Nikon Corp., Tokyo, Japan). The inhibition rate of cell migration was calculated as follows: inhibition rate of cell migration $=$ (number of migrated cells in the control group - number of migrated cells in the experimental group)/number of migrated cells in the control group $\times 100 \%$.

\section{In vitro endothelial cell tube formation assay}

After addition of about $150 \mu \mathrm{L} /$ well of liquid Matrigel (Corning Inc., Corning, NY, USA) to pre-cooled 48well plates with a pre-cooled pipette tip, the plates were placed on ice for $5 \mathrm{~min}$ to ensure Matrigel is evenly distributed across each well, followed by incubation at $37^{\circ} \mathrm{C}$ for $30 \mathrm{~min}$ to allow the gel to solidify. Endothelial cells were inoculated in the above 48-well plates at a density of $2 \times 10^{4}$ cells/well and supplemented with different concentrations of drugs diluted in complete culture medium to $200 \mu \mathrm{L}$, followed by incubation at $37^{\circ} \mathrm{C}$ for $8 \mathrm{~h}$. The formation of endothelial tubes in 5 randomly selected fields was observed and photographed under an inverted microscope (magnification 200x). The formed tube area in each field was analyzed by Image-J software. The inhibition rate of tube formation was calculated as follows: Inhibition rate of tube formation = (1 - the length of the lumen in the experimental group/the length of the lumen in the control group) $\times 100 \%$.

\section{Zebrafish embryonic-angiogenic assessment}


The flila-EGFP transgenic zebrafish with fluorescent blood vessels was purchased from the Model Animal Research Center of Nanjing University (Nanjing, China) [16]. Twenty-four hours after fertilization, zebrafish embryos were transferred to a 12-well plate containing 30 embryos/well and grouped for treatment as follows: Sev $(20 \mu \mathrm{g} / \mathrm{mL})+$ OXA $(20 \mu \mathrm{M}), \operatorname{Sev}(20 \mu \mathrm{g} / \mathrm{mL})+5-\mathrm{Fu}(20 \mu \mathrm{M}), \operatorname{Bev}(20 \mu \mathrm{g} / \mathrm{mL})+$ OXA $(20 \mu \mathrm{M}), \operatorname{Bev}(20 \mu \mathrm{g} / \mathrm{mL})+5-\mathrm{Fu}(20 \mu \mathrm{M})$, OXA $(20 \mu \mathrm{M}), 5-\mathrm{Fu}(20 \mu \mathrm{M}), \operatorname{Sev}(20 \mu \mathrm{g} / \mathrm{mL}), \operatorname{Bev}(20$ $\mu \mathrm{g} / \mathrm{mL}$ ), and cultured water (negative control). After continuous incubation at $28^{\circ} \mathrm{C}$ for $48 \mathrm{~h}$, the green fluorescence was excited under a fluorescence microscope with $450-490 \mathrm{~nm}$ wavelength blue light to observe and count the formation of intersegmental vessels (ISVs) of zebrafish embryos in the wells. The inhibition rate of ISVs was calculated as follows: inhibition rate of ISVs = (1 - the number of ISVs in the experimental group/the number of ISVs in the control group) $\times 100 \%$.

\section{Chick embryo chorioallantoic membrane (CAM) assay}

Specific pathogen-free (SPF) grade embryonated chicken eggs (White Leghorn) were purchased from Qianyuanhao Biological Co., Ltd. (Nanjing, China). A total of 108 9-day-old chicken embryos with similar body weights were randomly divided into the following 9 treatment groups containing 12 embryos in each group: Sev $(320 \mu \mathrm{g} / \mathrm{mL})+$ OXA $(20 \mu \mathrm{M})$, Sev $(320 \mu \mathrm{g} / \mathrm{mL})+5-\mathrm{Fu}(20 \mu \mathrm{M}), \operatorname{Bev}(320 \mu \mathrm{g} / \mathrm{mL})+$ OXA $(20 \mu \mathrm{M}), \operatorname{Bev}(320 \mu \mathrm{g} / \mathrm{mL})+5-\mathrm{Fu}(20 \mu \mathrm{M})$, OXA $(20 \mu \mathrm{M}), 5-\mathrm{Fu}(20 \mu \mathrm{M}), \operatorname{Sev}(320 \mu \mathrm{g} / \mathrm{mL}), \operatorname{Bev}(320 \mu \mathrm{g} / \mathrm{mL})$, and saline (negative control). The window at the end of the air chamber was opened under the egg light, and the eggshell and egg membrane were removed with ophthalmic tools to expose the allantoic tissue rich in blood vessels. A prepared methylcellulose dish containing $20 \mu \mathrm{L}$ of drugs was placed on the allantoic membrane of the chicken embryo. The window was then sealed with a sterile tape and returned to the incubator. After $72 \mathrm{~h}$, a mixture of methanol and acetone was added for fixation and blood vessel growth was observed and photographed under a dissecting microscope. The number of blood vessels in the field next to the methylcellulose dish was then calculated and compared.

\section{Tumor growth and angiogenesis evaluation in a mouse xenograft model}

A mouse xenograft model for HCC was established as described previously [15]. At the fourth generation, the tumor mass of HepG2-RFP or SMMC-7721 cells was inoculated into 65 mice. On day 10, there were 62 nude mice bearing SMMC-7721-xenografted tumors and 60 nude mice bearing HepG2-RFPxenografted tumors. Mice bearing HepG2-RFP- or SMMC-7721-xenografted tumors with satisfactory uniformity were then randomly divided into 9 groups ( $n=6$ mice for each group) as follows: Sev (5 $\mathrm{mg} / \mathrm{kg})+$ OXA (2 mg/kg), Sev (5 mg/kg) + 5-Fu (10 mg/kg), Sev (5 mg/kg), Bev (5 mg/kg) + OXA (2 $\mathrm{mg} / \mathrm{kg}), \operatorname{Bev}(5 \mathrm{mg} / \mathrm{kg})+5-\mathrm{Fu}(10 \mathrm{mg} / \mathrm{kg}), \operatorname{Bev}(5 \mathrm{mg} / \mathrm{kg})$, OXA (2 mg/kg), 5-Fu (10 mg/kg), and normal saline. After grouping, intraperitoneal injection into mice was performed twice a week for 3 weeks. As described previously [15], the volume and weight of SMMC-7721-xenografted tumors were monitored by physical measurement, while growth of HepG2-RFP-xenografted tumors and blood vessel density were assessed by an in vivo imaging system. 
The relative tumor volume (RTV) was calculated by $\mathrm{RTV}=\mathrm{Vt} / \mathrm{V} 0$, where $\mathrm{V} 0$ is the tumor volume prior to drug administration and $\mathrm{Vt}$ is the tumor volume measured at a given time following drug administration. Anti-tumor activity was evaluated by the relative tumor increase rate $(T / C)$ using the following formula: $(T / C \%)=$ TRTV/CRTV $\times 100$, where TRTV is the RTV of the treatment group and CRTV is the RTV of the negative control group. The therapeutic efficiency was evaluated based on the following criteria: T/C > $40 \%$ indicated no therapeutic effect, whereas $\mathrm{T} / \mathrm{C} \leq 40 \%$ with $\mathrm{P}<0.05$ indicated a positive therapeutic effect. Tumor weight inhibition rate (TWIR) was calculated as follows: TWIR = ( 1 - average tumor weight in the experimental group / mean tumor weight of control group) $\times 100 \%$.

\section{Histological analysis}

The histology of transplanted tumor specimens was observed by conventional hematoxylin and eosin (H\&E) staining and light microscopy. In addition, the expression levels of VEGF and CD31 were measured by immunohistochemistry, and the tumor tissue ultrastructure was observed by a transmission electron microscope (TEM; JEM-1011; JEOL, Ltd., Tokyo, Japan) as described previously [15].

\section{Statistical analysis}

All data were analyzed using SPSS v18.0 software (IBM, Armonk, NY, USA). Measured data were expressed as mean \pm standard deviation. Comparisons were made by $t$-test between the two groups and by one-way analysis of variance (ANOVA) among multiple groups. The immunohistochemical results were analyzed by $\chi^{2}$ test. $P<0.05$ was considered statistically significant.

\section{Results}

Sev and cytotoxic drugs synergistically induced cell growth inhibition, apoptosis and cell cycle arrest of $\mathrm{HCC} / \mathrm{endothelial}$ cells in vitro

The MTT assay showed that the growth of $3 \mathrm{HCC}$ cell lines and 3 endothelial cell lines was inhibited by the administration of different concentrations of Sev or Bev in combination with 5-Fu or OXA. As shown in Fig. 1A, compared with Bev + chemotherapy group at the same time point and same concentration, the Sev + chemotherapy group had a stronger inhibitory effect on the proliferation of 6 cell lines $(P<0.05)$. In addition, the results showed that after treatment for $48 \otimes 72 \mathrm{~h}$, the q values calculated by Jin's modified Bürgi's formula were all greater than 1 , indicating a synergistic interaction between Sev and chemotherapeutics.

In the present study, we used two different experimental methods to detect the drug-induced apoptosis. Analysis of morphological changes in the nuclei by 4',6-diamidino-2-phenylindole (DAPI) staining showed uniform, weak blue fluorescence and circular or elliptical nuclei in the negative control cells. In contrast, cells that were treated for $48 \mathrm{~h}$ in different groups displayed characteristic apoptotic nuclear morphological changes, including nuclear condensation and disintegration. 
Quantitative results revealed that the apoptosis rate of the 6 cell lines in the combined treatment group was markedly higher than that in the corresponding monotherapy group $(P<0.05)$. Moreover, the apoptosis rate in the Sev + chemotherapy group was significantly higher than that in the Bev + chemotherapy group ( $P<0.05$, Fig. 1B). To further confirm the above-mentioned results, we performed annexin- $\mathrm{V} /$ propidium iodide $(\mathrm{PI})$ double staining to determine the induced apoptosis. Compared with the negative control group, following $48 \mathrm{~h}$ of treatment, the apoptosis rate in the combined treatment group was notably higher than that in the corresponding monotherapy group $(P<0.05)$, and the apoptosis rate in the Sev + chemotherapy group was significantly higher than that in the Bev + chemotherapy group $(P<$ 0.05 , Fig. 1C).

The results of cell cycle detection by flow cytometry showed that compared with the negative control group, the proportion of HepG2, Bel-7402, HUVEC, and HMEC-1 cells in the S phase as well as SMMC7721 and EAhy 92 cells in the G0/G1 phase increased in each combination treatment group $(P<0.05)$, indicating that the combined medication induced growth cycle arrest in $3 \mathrm{HCC}$ cells and 3 endothelial cell lines (Fig. 1D). Compared with Bev, the combination of Sev with chemotherapeutics more effectively induced growth cycle arrest in HepG2, SMMC-7721, HUVEC, and EAhy92 cells $(P<0.05)$.

\section{Sev in combination with cytotoxic drugs enhanced the anti-proliferation effects and induced apoptosis in mouse HCC xenografts}

To investigate the in vivo anti-proliferation effects of combination therapy, we monitored the tumor growth of HepG2-RFP or SMMC-7721-xenografted tumors in different groups using a fluorescence imaging system or the measurement with Vernier caliper respectively. As illustrated in Fig. 2A, at the end of drug administration, the RTVs of both HepG2-RFP- and SMMC-7721-xenografted tumors in each treatment group were significantly lower than those in the negative control group $(P<0.05)$. Compared with the corresponding monotherapy, the combined drugs had a stronger inhibitory effect on the growth of HepG2-RFP- and SMMC-7721-xenografted tumors. In addition, the combination of Sev with chemotherapy showed a stronger inhibitory influence on the growth of xenografted tumors than Bev + chemotherapy $(P<0.05)$.

H\&E staining demonstrated that 21 days after drug administration in the HepG2-RFP- and SMMC-7721xenografted tumors without drug treatment, the tumor tissue structure was compact with less interstitial fibers, the tumor cells were pleomorphic spindle-shaped with a large volume of tumor cells and deeply stained nucleus, and there were a large number of mitotic cells with less cytoplasm. The tumor tissue in the OXA/5-FU single-drug group showed patchy necrosis with scattered apoptotic cells. In the Sev and Bev monotherapy groups, the majority of tumor tissues were still in a good structure, with surrounded patchy necrosis and apoptosis. In contrast, in each combination group, the area of necrotic tumor cells was significantly increased, with surrounding patches of apoptotic tumor cells (Fig. 2B).

At 21 days after drug administration, TEM of tumors in the negative control group showed an intact cell membrane, abundant microvilli, normal organelle structure, large and multiple nucleoli, uniform chromatin, and enriched euchromatin. In contrast, apparent cell apoptosis was observed in each 
treatment group as demonstrated by cell shrinkage, cytoplasmic condensation, chromatin condensation, and rupture or chromatin condensation on the inside of the nuclear membrane, increase of cytoplasmic electron density, formation of a blistering cytoplasm and apoptotic body, mitochondrial swelling, vacuolization, and reduction or disappearance of cell surface microvilli. In addition, more apoptotic cells were found in the combination groups of Sev and Bev, compared with the monotherapy groups (Fig. 2C).

\section{Sev combined with chemotherapeutics inhibited migration and tube formation of endothelial cells in vitro}

In this study, both wound healing and transwell migration assays were used to detect the therapeutic effects on the migration of HUVEC, EAhy926, and HMEC-1 cells. In wound healing assay,after $24 \mathrm{~h}$ of drug administration into 3 endothelial cell lines, compared with the negative control group and the corresponding monotherapy group, the degree of wound closure in each combination group was markedly reduced $(P<0.05$, Fig. $4 \mathrm{~A})$. In addition, the degree of wound closure was also lower in the Sev + chemotherapy group than in the Bev + chemotherapy group $(P<0.05$, Fig. $3 A)$. In the transwell migration assay, quantitative analysis results showed that compared with the negative control group and the corresponding monotherapy group, the inhibition rate of cell migration in each combination group was significantly higher $(P<0.05)$. Meanwhile, the mentioned rate was higher in the Sev + chemotherapy group than that in the Bev + chemotherapy group $(P<0.05$, Fig. 3B). These results suggested that the migration of endothelial cells was inhibited more in the Sev + 5-Fu/OXA group than in the Bev + chemotherapy group, which is consistent with the results of wound healing assay.

The development of capillary tubes and sprouting of new capillaries are hallmarks of angiogenesis during solid tumor growth. To evaluate the therapeutic effects on this reorganization stage during angiogenesis, in vitro tube formation assay was performed. As displayed in Fig. 4C, the arrangement of the tubes in the negative control group was essentially complete, while that in the treatment group was mostly incomplete and had a sparse network. The results showed that compared with the negative control group and the corresponding monotherapy group, the inhibition rate of tube formation in each combination group was noticeably higher $(P<0.05)$. Meanwhile, the mentioned rate was higher in the Sev + chemotherapy group than that in the Bev + chemotherapy group $(P<0.05$, Fig. $3 C)$. These results demonstrated that the tube formation of human endothelial cells was inhibited more in the Sev +5Fu/OXA group than in the Bev + chemotherapy group.

\section{Anti-angiogenic effects of Sev in combination with chemotherapy in zebrafish, chicken embryos, and mouse HCC xenografts}

As shown in Fig. 4A,compared with the negative control group, zebrafish embryo ISV in all treatment groups displayed morphological abnormalities or deletions. Compared with the negative control group and the corresponding monotherapy group, the inhibition rate of ISV formation in each combination group was significantly enhanced $(P<0.05)$. In addition, that rate was also higher in the Sev + chemotherapy group than in the Bev + chemotherapy group $(P<0.05)$. The quantitative reverse transcription polymerase chain reaction (RT-qPCR) testing of zebrafish embryonic tissue showed that the downregulation of VEGF mRNA level was greater in the combination treatment group than that in the 
corresponding monotherapy group. Compared with Bev + chemotherapy group, the VEGF mRNA level in the Sev + chemotherapy group was markedly lower $(P<0.0$, Fig. 4B).

As depicted in Fig. 4C, the results of the CAM angiogenesis experiment showed that (compared with the negative control group) the growth of CAM blood vessels in each treatment group was impaired with thin vessel diameter, reduced density, lighter color, or partial loss of vessels. Moreover, the anti-angiogenic effects in CAM were stronger in the combination treatment group than those in the corresponding monotherapy group and greater in the Sev + chemotherapy group than that in the Bev + chemotherapy group $(P<0.05)$.

In the present study, a fluorescence imaging system was applied to observe the growth of blood vessels in HepG2-RFP-xenografted tumors. At 3 weeks after drug administration, the tumor vascular density was significantly decreased in the treatment groups compared with that in the negative control group $(P<$ 0.05). Moreover, the tumor vascular density was notably lower in the combination treatment groups than that in the monotherapy groups, as well as being lower in the Sev + chemotherapy group than that in the Bev + chemotherapy group $(P<0.05)$ (Fig. 4D). Further detection of MVD by immunohistochemical analysis of CD31 showed that there was a significantly decreased MVD expression in the treatment groups at 3 weeks after administration $(P<0.05)$. In addition, the MVD expression was remarkably downregulated in the combination treatment groups than that in the monotherapy groups, while the MVD in the Sev + chemotherapy group was lower than that in the Bev + chemotherapy group $(P<0.05)$ (Fig. 4E).

\section{Sev combined with chemotherapeutics downregulated the VEGF/VEGFR expression both in vitro and in vivo}

The results of ELISA demonstrated that in comparison with the control group, treatments significantly reduced the VEGF levels in the cell culture medium in the 6 cell lines $(P<0.05)$. Moreover, the reduction of VEGF levels was greater in the combination therapy groups than that in the monotherapy groups $(P<$ 0.05). In the meantime, the reduction of VEGF levels in the Sev + chemotherapy group was significantly stronger than that in the Bev + chemotherapy group $(P<0.05)($ Fig. $5 \mathrm{~A})$.

The results of Western blotting of cultured cells showed that compared with monotherapy, treatment with Sev or Bev in combination with OXA/5-Fu for $48 \mathrm{~h}$ downregulated the expression levels of VEGF, $\mathrm{p}$ VEGFR1, or p-VEGFR2 in the 6 cell lines and this effect was stronger in Sev + chemotherapy group than that in Bev + chemotherapy group ( $P<0.05$, Fig. 5B). Similarly, Western blotting of tumor tissues revealed that there were high expression levels of VEGF and VEGFR1 in HepG2-RFP- and SMMC-7721-xenografted tumors, and VEGFR2 was highly expressed in HepG2-RFP-xenografted tumors while being undetectable in SMMC-7721-xenografted tumors. After treatment with Sev or Bev in combination with OXA/5-Fu, the expression levels of VEGF, p-VEGFR1, or p-VEGFR2 were suppressed in both HepG2-RFP- and SMMC7721-xenografted tumors ( $P<0.05$, Fig. 5C). 


\section{Discussion}

HCC has historically been considered resistant to common chemotherapy until several high-efficiency drugs with low-toxicity, e.g. OXA, were developed and used clinically in the last two decades [17]. OXA is a platinum-based cytotoxic agent with an activity against several cisplatin-resistant cell lines and solid tumors. In addition, the combination of OXA with 5-Fu was found to have synergistic anti-proliferative effects on several tumor models[18]. The promising results of OXA in advanced HCC have also been documented in a series of clinical trials [19]. Particularly, in March 2013, the FOLFOX4 regimen (OXA, 5$\mathrm{Fu}$, and leucovorin) for advanced HCC patients was officially approved in China [20]. Although the traditional concept of the ineffectiveness of systemic chemotherapy for HCC was subverted, it has also been suggested that combination therapy with different mechanisms may provide more promising antitumor effects due to the complexity of the pathogenesis of HCC[17].

Previous studies have confirmed that anti-VEGF targeted therapy and chemotherapy have synergistic anti-tumor effects [21]. Jain et al. pointed out that anti-angiogenesis therapy can reduce interstitial pressure by normalizing tumor blood vessels, thereby promoting the absorption of chemotherapeutics by tumor tissues, reducing the resistance of tumor to chemotherapy, and improving the efficacy of chemotherapy [22]. Klement et al. demonstrated that chemotherapy (especially low-dose chemotherapy) can cause damage to endothelial cells in advance, and when the important survival factor of endothelial cells-VEGF-is simultaneously blocked, the cytotoxic effects of chemotherapy on tumor cells can be enhanced [23]. Shi et al. reported that anti-VEGF antibody could reverse the resistance of SMMC-7721 to the cytotoxic drug etoposide, thus indicating that anti-VEGF antibody combined with etoposide has a synergistic anti-HCC effect [24].

According to in vivo and in vitro studies, Katsura et al. found that VEGFR inhibitors IFN and 5-Fu may synergistically inhibit the proliferation of HCC and HUVEC cells and induce apoptosis. They also demonstrated that the synergistic mechanism could be related to inhibition of the VEG/VEGFR signaling pathway [25]. Two other studies also found that because cytotoxic chemotherapy can inhibit proliferation and migration of endothelial cells, it can also be used as an anti-angiogenic agent in combination with targeted-angiogenic inhibitors to produce synergistic effects [26, 27].

Sev is a monoclonal anti-VEGF antibody (mAb) with the same degree of humanization as Bev. Sev and Bev are both IgG1-type antibodies with similar physical and chemical properties and the same target. Nevertheless, these two mAbs are distinct in the CDR sequences. Our previous research found that by targeting the VEGF/VEGFR pathway of HCC cells through the autocrine pathway, Sev can inhibit the phosphorylation levels of ERK1/2 and AKT proteins and regulate the downstream activities of Caspase-3, PRAP, p21, Rb, and Cycline D1, resulting in cell cycle arrest, cell apoptosis, and inhibition to the growth of HCC cells. We also found that Sev can target VEGF/VEGFR pathway in endothelial cells, thereby inhibiting the phosphorylation levels of ERK1/2 and AKT proteins, promoting cell cycle arrest, inducing cell apoptosis, as well as inhibiting the proliferation, migration, and vascular formation of endothelial cells. 
Our findings showed that Sev has greater anti-HCC cell growth and anti-angiogenic effects than Bev in both cellular and animal models [15].

In the current research, we used cellular and animal models to explore the influences of Sev in combination with OXA/5-Fu on growth and angiogenesis of HCC cells, and to compare with the Bev. In vitro results showed that the combination of Sev or Bev with OXA/5-Fu at different concentrations can synergistically inhibit the proliferation of $\mathrm{HCC}$ and endothelial cells. Compared with the corresponding monotherapy group, combination therapy also showed a stronger effect on inducing apoptosis and cell cycle arrest in the S phase. In vivo findings revealed that Sev in combination with chemotherapy can synergistically inhibit the tumor growth by inducing cell apoptosis in nude mice with HCC xenografts. In addition, the cell scratch experiment and the transwell co-culture model revealed that Sev in combination with chemotherapy can inhibit the migration of endothelial cell lines. The tube formation test, zebrafish and chicken embryonic angiogenesis studies, immunohistochemistry, and in vivo fluorescence imaging consistently verified that Sev combined with OXA/5-Fu can synergistically inhibit the growth of blood vessels, and the underlying mechanism may be associated with inhibition of the VEGF/VEGFR signaling pathway. In addition, OXA/5-Fu combined with Sev showed stronger anti-proliferation and antiangiogenic effects than combination with Bev.

\section{Conclusion}

The present preclinical study revealed that both $\mathrm{HCC} /$ endothelial cell lines and mice xenografts treated with Sev in combination with OXA/5-Fu were accompanied by less HCC cell growth and angiogenesis than those treated with control or single agents. In addition to the VEGF blockade mediated by Sev, cytotoxicity triggered by OXA/5-Fu against either cancer cells or endothelial cells may contribute to the synergistic effects of anti-proliferation and anti-angiogenic on HCC cells.

Our findings may provide experimental evidence and theoretical biological rationale for assessing combination therapy with Sev and OXA/5-Fu as a promising regimen in future clinical settings of HCC treatment.

\section{Abbreviations}

HCC, Hepatocellular carcinoma; VEGF, vascular endothelial growth factor; VEGFR, vascular endothelial growth factor receptor; Bev, Bevacizumab; RECIST, Response Evaluation Criteria in Solid Tumors; OXA, oxaliplatin; Sev, Sevacizumab; 5-Fu, 5-fluorouracil; HUVECs, Human umbilical vein endothelial cells; ELISA, enzyme-linked immunosorbent assay; RT-qPCR, quantitative reverse transcription polymerase chain reaction; PBS, phosphate-buffered saline; CAM, chick embryo chorioallantoic membrane; MVD, tumor tissue microvessel density; RTV, relative tumor volume

\section{Declarations}


Acknowledgements

The authors thank Jiangsu Simcere Pharmaceutical Co., Ltd. (Nanjing, China) for the gift of sevacizumab.

\section{Authors' contributions}

LL and SQ conceived the experiments. LL, LH, MZ, NL, ZL and YG performed the experiments, wrote the manuscript, and prepared the figures. YZ and NG Zhang Yu carried out experiment quality control. All authors read and approved the final manuscript.

\section{Funding}

This study was funded by grants from the National Key R \& D Program of China (No. 2017YFA0205502), the CSCO Cancer Research Fund (No. Y-HR2018-151) and the Science \& Technology Support Program of Jiangsu Province (No. BE2012766).

\section{Availability of data and materials}

All the data and materials were available under the agreement of the authors.

\section{Ethics approval and consent to participate}

All experimental procedures involving animals and their care were conducted in conformity with People's Republic of China State Council Regulations for Laboratory Animal Management (enacted in 1988) and were approved by Animal Care and Use Committee of Southeast University, Nanjing, China.

\section{Consent for publication}

Not applicable.

\section{Competing interests}

The authors declare that they have no competing interests.

\section{References}

1. Gong J, Chuang J, Cho M, Toomey K, Hendifar A, Li D. Molecular targets, pathways, and therapeutic ilmplications for hepatocellular carcinoma. Int J Mol Sci. 2020;21(15):5232.

2. Zhan P, Qian Q, Yu LK. Prognostic significance of vascular endothelial growth factor expression in hepatocellular carcinoma tissue: a meta-analysis. Hepatobiliary Surgery Nutrition. 2013;2(3):148-55.

3. Huang J, Zhang X, Tang Q, Zhang F, Li Y, Feng Z, Zhu J. Prognostic significance and potential therapeutic target of VEGFR2 in hepatocellular carcinoma. J Clin Pathol. 2011;64(4):343-8. 
4. Morse MA, Sun W, Kim R, He AR, Abada PB, Mynderse M, Finn RS. The role of angiogenesis in hepatocellular carcinoma. Clin Cancer Res. 2019;25(3):912-20. https://doi.org/10.1158/10780432.CCR-18-1254.

5. Ferrara N, Adamis AP. Ten years of anti-vascular endothelial growth factor therapy. Nat Rev Drug Discovery. 2016;15(6):385-403.

6. Al-Abd AM, Alamoudi AJ, Abdel-Naim AB, Neamatallah TA, Ashour OM. Anti-angiogenic agents for the treatment of solid tumors: Potential pathways, therapy and current strategies - A review. J Adv Res. 2017;8(6):591-605.

7. Rajabi M, Mousa SA. The role of angiogenesis in cancer treatment. Biomedicines. 2017;5(2):34.

8. Siegel AB, Cohen El, Ocean A, Lehrer D, Goldenberg A, Knox JJ, Chen H, Clark-Garvey S, Weinberg A, Mandeli J, Christos P, Mazumdar M, Popa E, Brown R, Rafii S, Schwartz JD. Phase II trial evaluating the clinical and biologic effects of bevacizumab in unresectable hepatocellular carcinoma. J Clin Oncol. 2008;26(18):2992-98.

9. Garcia J, Hurwitz HI, Sandler AB, David M, Coleman RL, Deurloo R, Chinot OL. Bevacizumab (Avastin $\left.{ }^{\circledR}\right)$ in cancer treatment: A review of 15 years of clinical experience and future outlook. Cancer Treat Rev. 2020;86:102017.

10. Ferrara N, Hillan KJ, Gerber HP, Novotny W. Discovery and development of bevacizumab, an antiVEGF antibody for treating cancer. Nat Rev Drug Discovery. 2004;3(5):391-400.

11. Fang P, Hu J, Cheng Z, Liu Z, Wang J, Jiao S. Efficacy and safety of bevacizumab for the treatment of advanced hepatocellular carcinoma: a systematic review of phase II trials. PLoS One. 2012;7(12):e49717.

12. Hsu CH, Yang TS, Hsu C, Toh HC, Epstein RJ, Hsiao LT, Chen PJ, Lin ZZ, Chao TY, Cheng AL. Efficacy and tolerability of bevacizumab plus capecitabine as first-line therapy in patients with advanced hepatocellular carcinoma. Br J Cancer. 2010;102(6):981-6.

13. Zhu AX, Blaszkowsky LS, Ryan DP, Clark JW, Muzikansky A, Horgan K, Sheehan S, Hale KE, Enzinger PC, Bhargava P, Stuart K. Phase II study of gemcitabine and oxaliplatin in combination with bevacizumab in patients with advanced hepatocellular carcinoma. J Clin Oncol. 2006;24(12):1898903.

14. Sun W, Sohal D, Haller DG, Mykulowycz K, Rosen M, Soulen MC, Caparro M, Teitelbaum UR, Giantonio B. O'Dwyer PJ,Shaked A, Reddy R, Olthoff K. Phase 2 trial of bevacizumab, capecitabine, and oxaliplatin in treatment of advanced hepatocellular carcinoma. Cancer. 2011; 117(14):3187-92.

15. Liu L, Qin S, Zheng Y, Han L, Zhang M, Luo N, Liu Z, Gu N, Gu X, Yin X. Molecular targeting of VEGF/VEGFR signaling by the anti-VEGF monoclonal antibody BD0801 inhibits the growth and induces apoptosis of human hepatocellular carcinoma cells in vitro and in vivo. Cancer Biol Ther. 2017;18(3):166-76.

16. Lawson ND, Weinstein BM. In vivo imaging of embryonic vascular development using transgenic zebrafish. Dev Biol. 2002;248(2):307-18. 
17. Gong X, Qin S. Progress in systemic therapy of advanced hepatocellular carcinoma. World J Gastroenterol. 2016;22(29):6582-94.

18. Raymond E, Chaney SG, Taamma A, Cvitkovic E. Oxaliplatin. A review of preclinical and clinical studies. Ann Oncol. 1998;9(10):1053-71.

19. Petrelli F, Coinu A, Borgonovo K, Cabiddu M, Ghilardi M, Lonati V, Barni S. Oxaliplatin-based chemotherapy: a new option in advanced hepatocellular carcinoma. a systematic review and pooled analysis. Clin Oncol (R Coll Radiol). 2014;26(8):488-96.

20. Qin S, Bai Y, Lim HY, Thongprasert S, Chao Y, Fan J, Yang TS, Bhudhisawasdi V, Kang WK, Zhou Y, Lee JH, sun Y. Randomized, multicenter, open-label study of oxaliplatin plus fluorouracil/leucovorin versus doxorubicin as palliative chemotherapy in patients with advanced hepatocellular carcinoma from Asia. Journal of Clinical Oncology. 2013; 31 (28):3501-8.

21. Roderburg C, Özdirik B, Wree A, Demir M, Tacke F. Systemic treatment of hepatocellular carcinoma: from sorafenib to combination therapies. Hepatology Oncology. 2020;7(2):HEP20.

22. Jain RK. Normalizing tumor vasculature with antiangiogenic therapy:a new paradigm for combination therapy. Nat Med. 2001;7(9):987-9.

23. Klement G, Baruchel S, Rak J, Man S, Clark K, Hicklin DJ, Bohlen P, Kerbel RS. Continuous low-dose therapy with vinblastine and VEGF receptor-2 antibody induces sustained tumor regression without overt toxicity. Journal of Clinical Investigation. 2000;105(8):R15-24.

24. Shi S, Yuan C, Zhuang K, Liang G, Yao Z, Wang D, Weng Q, Cao J, Luo P, Zhu H, Ding L, Ma S. Resistance of SMMC-7721 hepatoma cells to etoposide in hypoxia is reversed by VEGF inhibitor. Mol Med Rep. 2015;11(5):3842-47.

25. Katsura Y, Wada H, Murakami M, Akita H, Hama N, Kawamoto K, Kobayashi S, Marubashi S, Eguchi H, Tanemura M, Umeshita K, Doki Y, Mori M, Nagano H. PTK787/ZK222584 combined with interfern alpha and 5-fluorouracil synergistically inhibits VEGF signaling pathway in hepatocellular carcinoma. Annals of Surgical Oncology. 2013; Suppl 3:S517-26.

26. Wang J, Lou P, Lesniewski R, Henkin J. Paclitaxel at ultra concentrations inhibits angiogenesis without affecting cellular microtubule assembly. Anticancer Drugs. 2003;14(1):13-9.

27. Sweeney CJ, Miller KD, Sissons SE, Nozaki S, Heilman DK, Shen J, Sledge GW Jr. The antiangiogenic property of docetaxel is synergistic with a recombinant humanized monoclonal antibody against vascular endothelial growth factor or 2-methoxyestradiol but antagonized by endothelial growth factors. Can Res. 2001;61(8):3369-72.

\section{Figures}



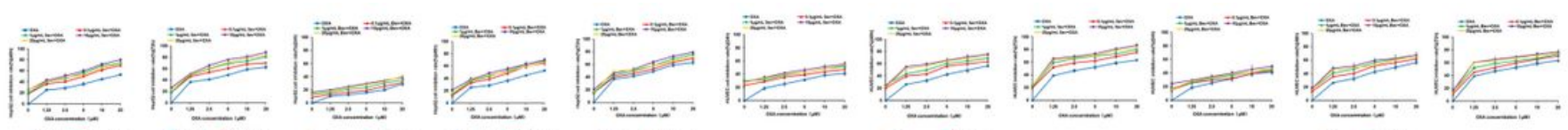

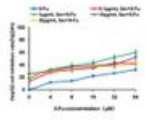
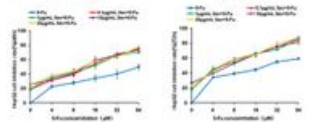

:
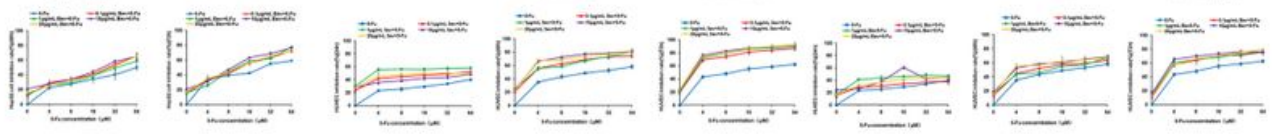

$=$
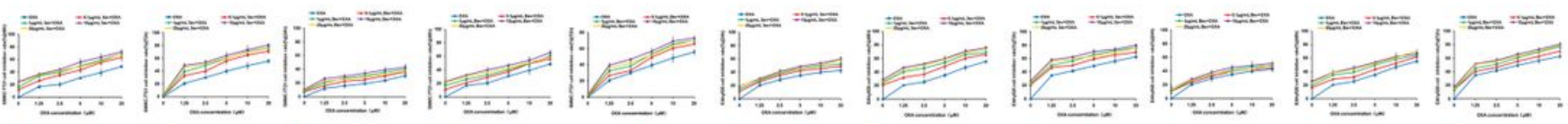

$\mid$
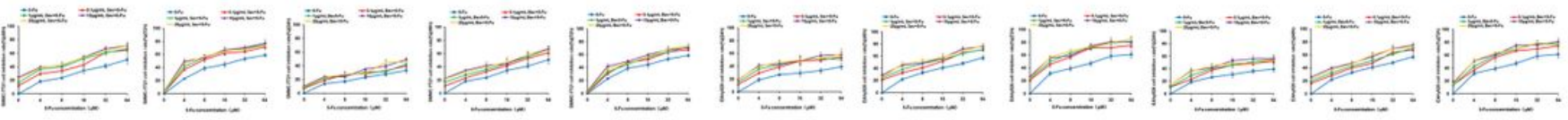

$\sum_{n=1}^{=-1}$
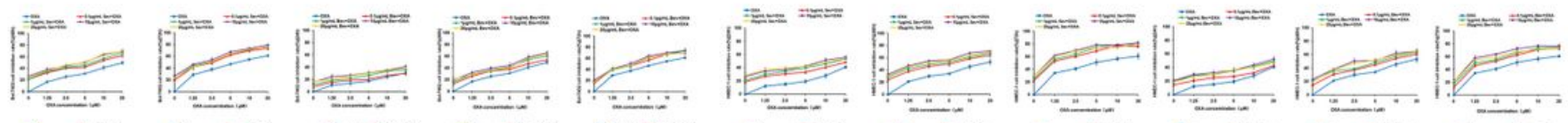

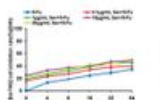
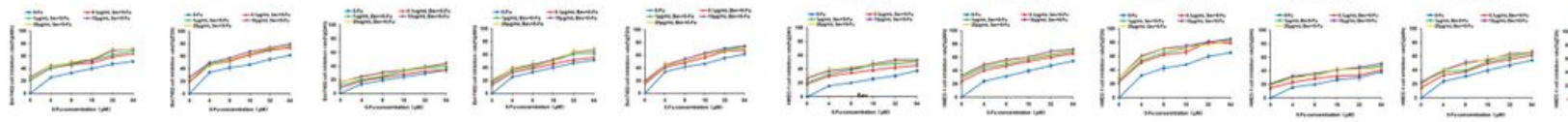

:
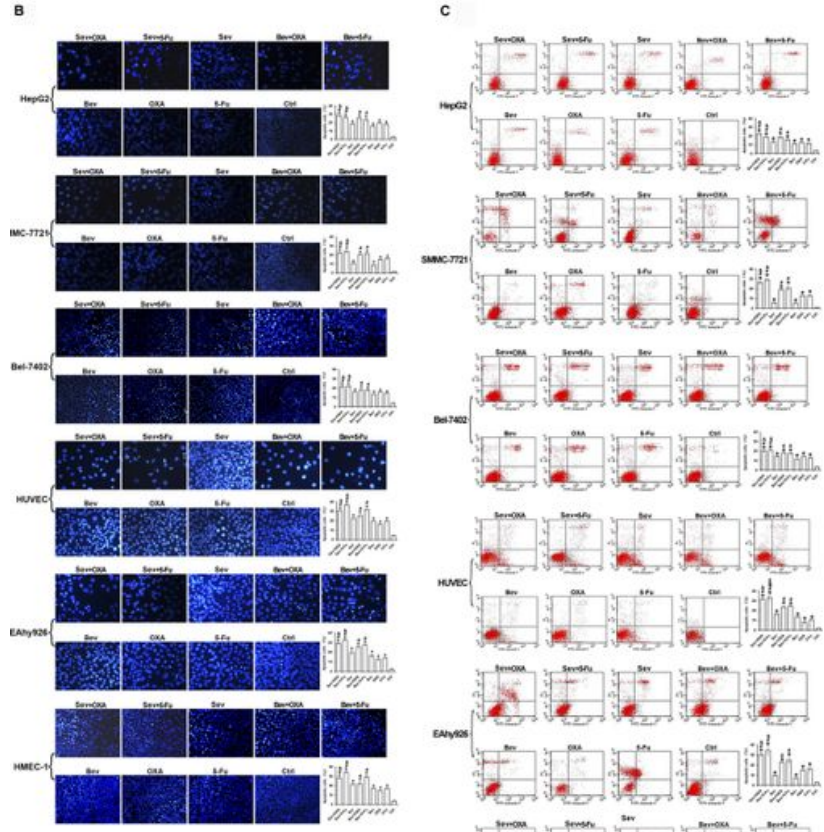

D
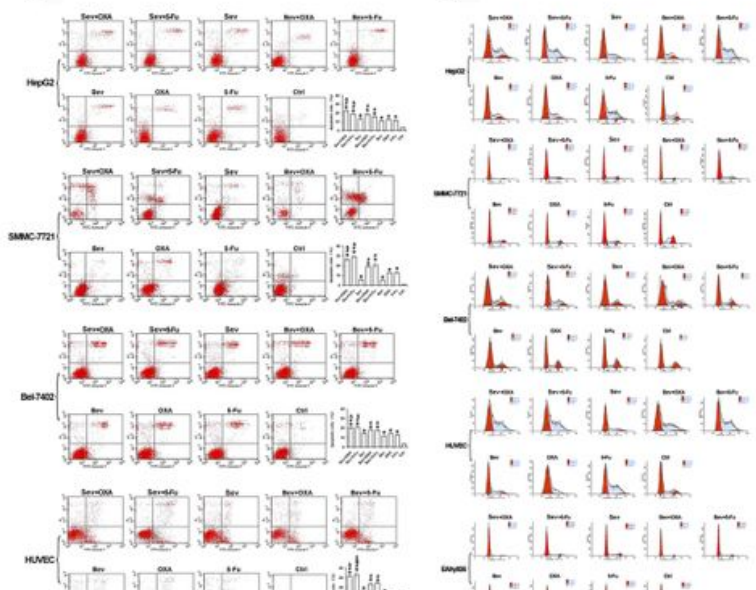

TISTL

["L"L" L" L"

IELE

IL LE L L

IELE

ILIIL:

$\left[L^{2} L^{-} L^{-} L^{\circ}\right.$

$-L^{-} L^{\prime} L^{\circ}$

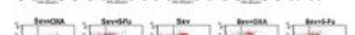

$-I E L E$

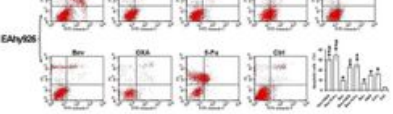

[C I'L

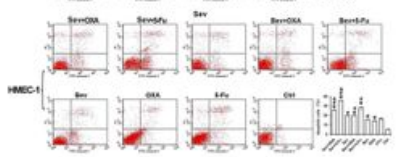

\section{Figure 1}

Sev and cytotoxic drugs synergistically inhibited the growth of of human HCC/endothelial cells in vitro. The HCC cell lines (HepG2, SMMC-7721, and Bel7402) and endothelial cells (HUVEC, EAhy926, and HMEC-1) were treated with the different concentrations of Sev or Bev in combination with 5-Fu or OXA for 24,48 , and $72 \mathrm{~h}$, respectively. (A) The cell viability was assessed by the MTT assay. The mean inhibition rate \pm standard deviation was obtained from four independent experiments. (B) Cell apoptosis was analyzed by DAPI staining (left panels) of the indicated cells (magnification, 200x). Right panels, statistical data of the apoptosis rate ( ${ }^{*} \mathrm{P}<0.05$, compared with the control group; \# $\mathrm{P}<0.05$, compared with the corresponding monotherapy groups; $\& \mathrm{P}<0.05$, compared with the Bev + chemotherapy groups). 
(C) Cell apoptosis was analyzed by annexin V/PI staining (left panels). The lower left quadrant: the double-negative viable cells; the lower right quadrant: apoptotic cells with double annexin V+/PI- staining; the upper right quadrant: the late apoptotic/necrotic cells with annexin V+/PI+ staining. Right panels, statistical data of the apoptosis rate ( ${ }^{*} P<0.05$, compared with the control group; $\# P<0.05$, compared with the corresponding monotherapy groups; \& $\mathrm{P}<0.05$, compared with the Bev + chemotherapy groups). (D) Six cell lines were treated with different concentrations of Sev or Bev in combination with 5-Fu or OXA for $48 \mathrm{~h}$. The cell cycle distribution was determined by PI staining coupled with flow cytometry. Statistical data was obtained from the results of four repeated experiments. 
A
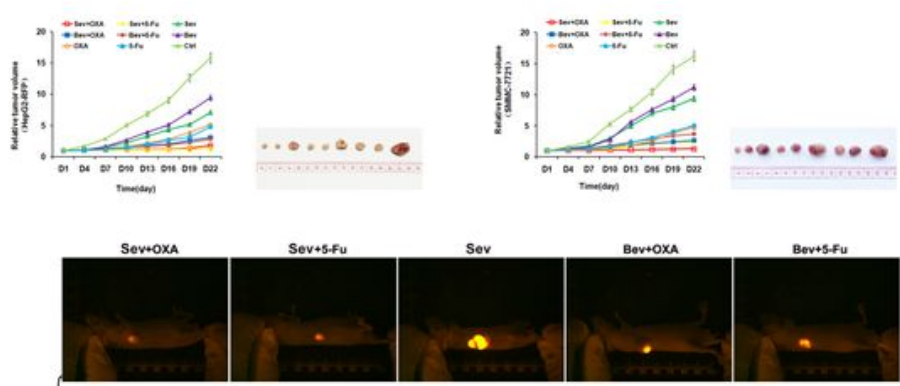

HepG2-RFP

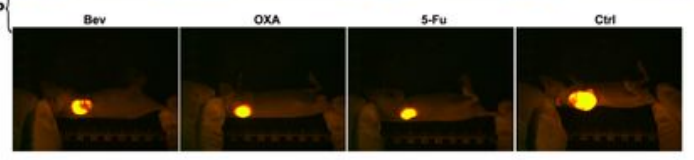

B

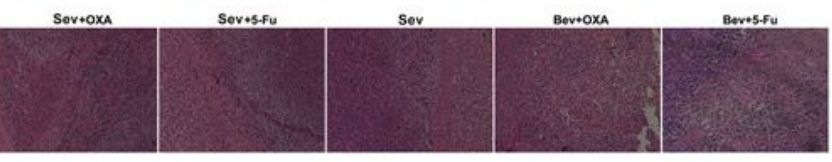

HepG2-RFP
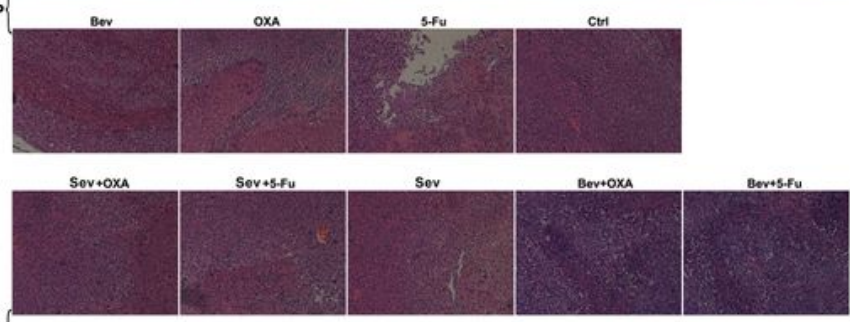

SMMC-7721

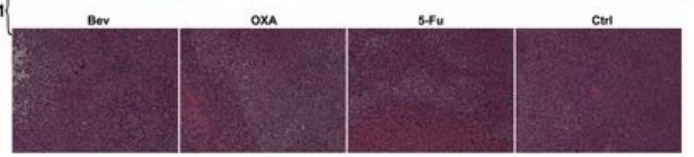

C

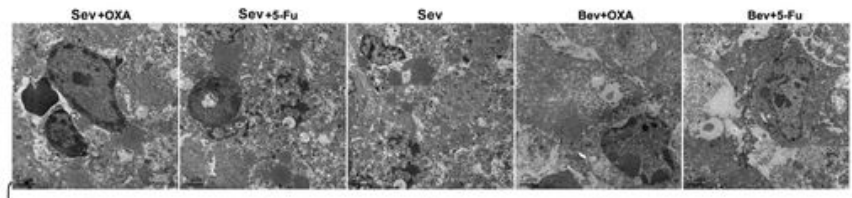

HepG2-RFP
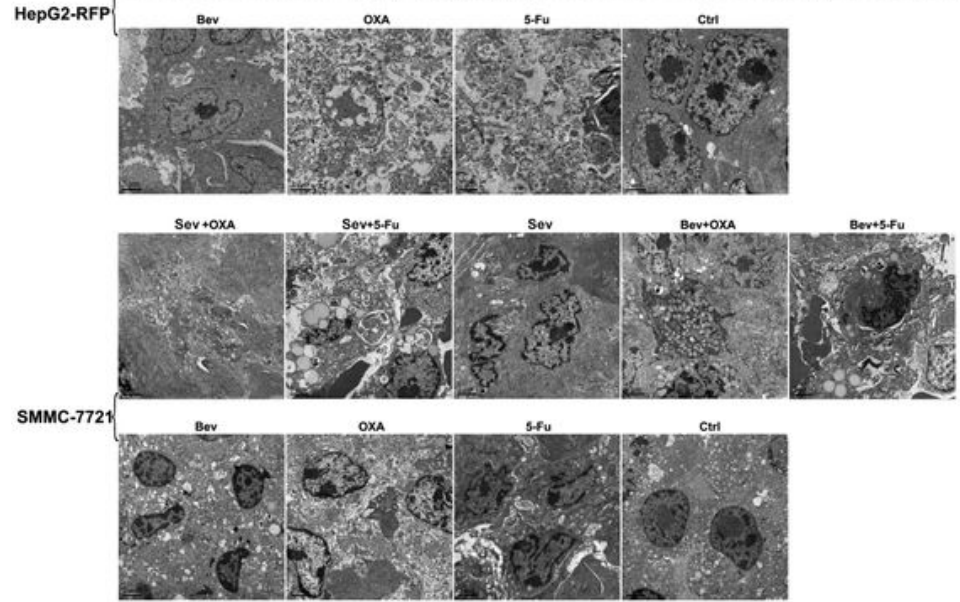

Figure 2

Sev in combination with cytotoxic drugs enhanced the anti-proliferation effects in mouse HCC xenografts. (A) Mice bearing HepG2-RFP or SMMC-7721 cell xenografts were administered with different doses of Sev or Bev in combination with 5-Fu or OXA. The growth of tumors was monitored using a fluorescence imaging system and a vernier caliper measurement, respectively. (B) H\&E staining of representative tumor xenografts from mice following treatment with the indicated drugs. Sev-induced 
necrosis with apparent cell apoptosis (magnification 200x). (C) Apoptotic cells of tumor xenografts in indicated treatment groups were determined by TEM.
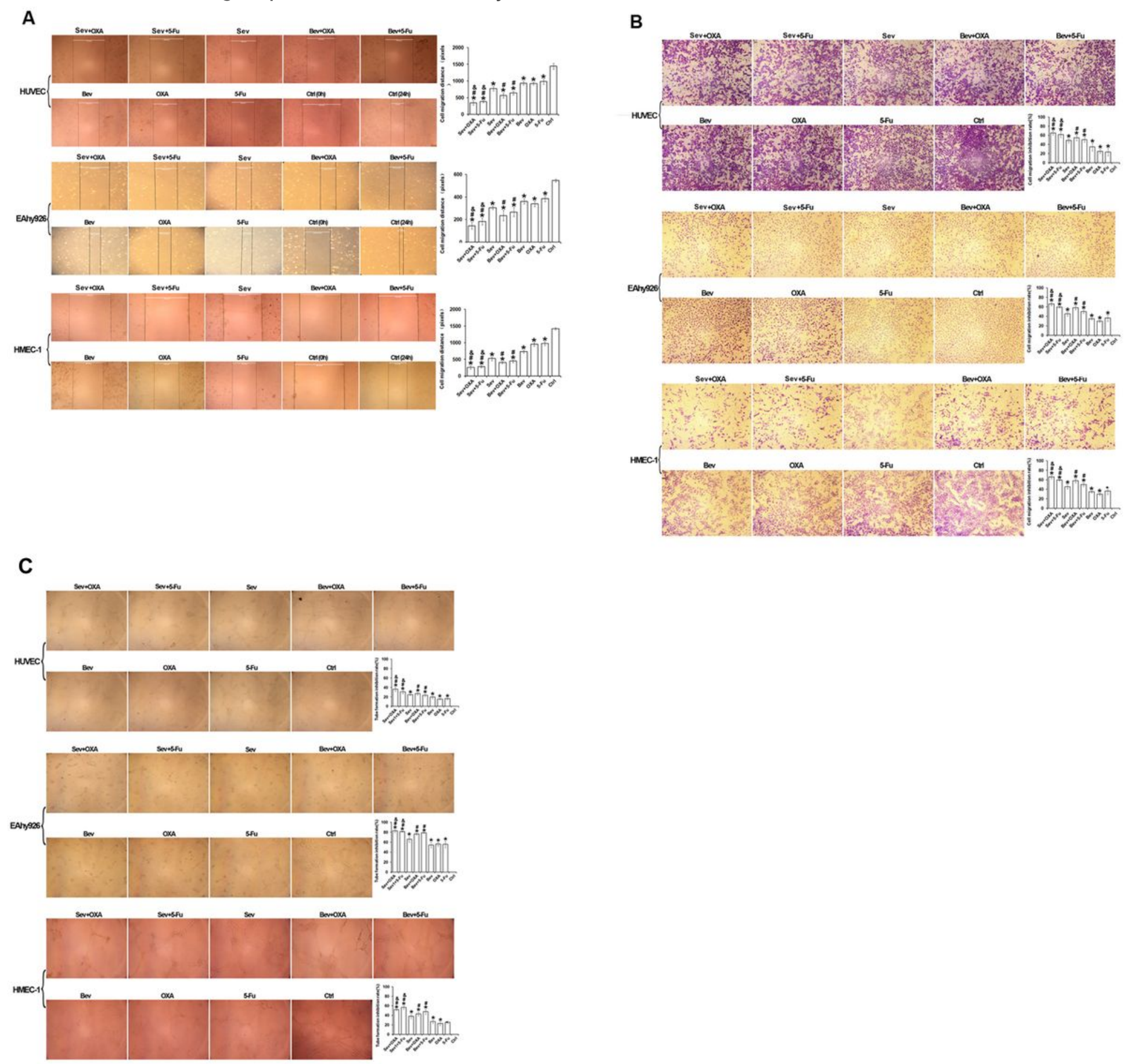

\section{Figure 3}

Sev combined with chemotherapeutics inhibited migration and tube formation of endothelial cells in vitro. (A) Wound healing assay for detecting the therapeutic effects on the migration of 3 endothelial cell lines (HUVEC, EAhy926, and HMEC-1 cells). After $24 \mathrm{~h}$ of drug administration, the degree of wound closure in Sev combined with 5-Fu or OXA groups was markedly reduced ( ${ }^{\mathrm{P}}<0.05$, compared with the control group; \# $\mathrm{P}<0.05$, compared with the corresponding monotherapy groups; \& $\mathrm{P}<0.05$, compared with the Bev + chemotherapy groups). (B) Transwell migration assays for detecting the inhibition effects 
on the migration of 3 endothelial cell lines. Quantitative analysis results showed that the inhibition rate of cell migration in Sev combined with 5-Fu or OXA groups was significantly higher $\left({ }^{\star} \mathrm{P}<0.05\right.$, compared with the control group; \# $\mathrm{P}<0.05$, compared with the corresponding monotherapy groups; \& $\mathrm{P}<0.05$, compared with the Bev + chemotherapy groups). (C) In vitro tube formation assay for evaluating antiangiogenesis effect of drugs. After $8 \mathrm{~h}$ of drug administration, the inhibition rate of tube formation in Sev combined with 5-Fu or OXA groups was noticeably higher ( ${ }^{\star} \mathrm{P}<0.05$, compared with the control group; \# $\mathrm{P}<0.05$, compared with the corresponding monotherapy groups; \& $\mathrm{P}<0.05$, compared with the Bev + chemotherapy groups). 
A

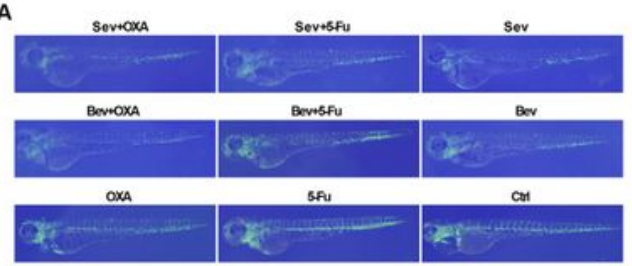

C
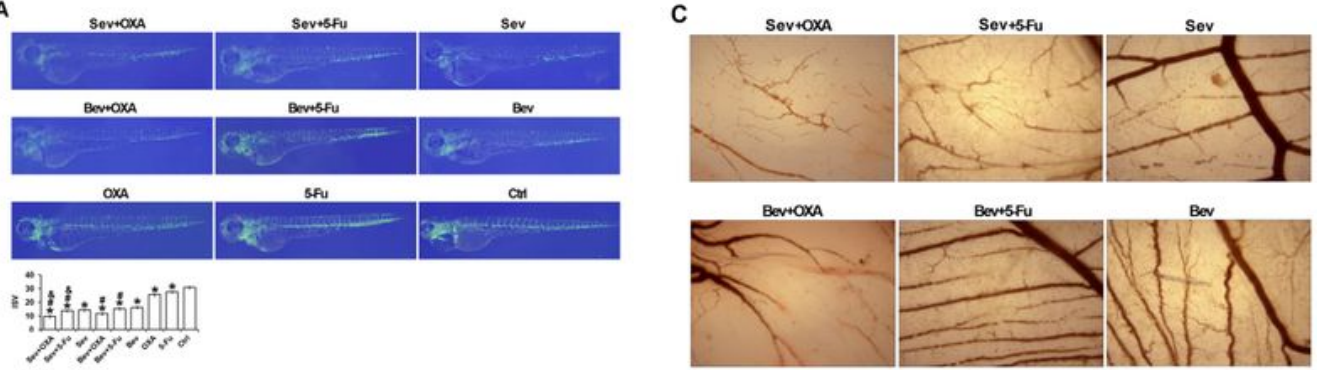

B
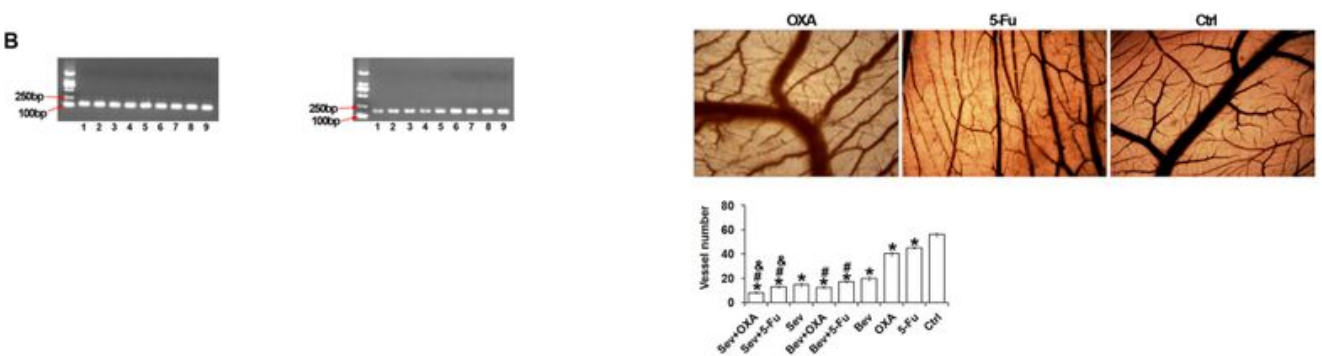

D
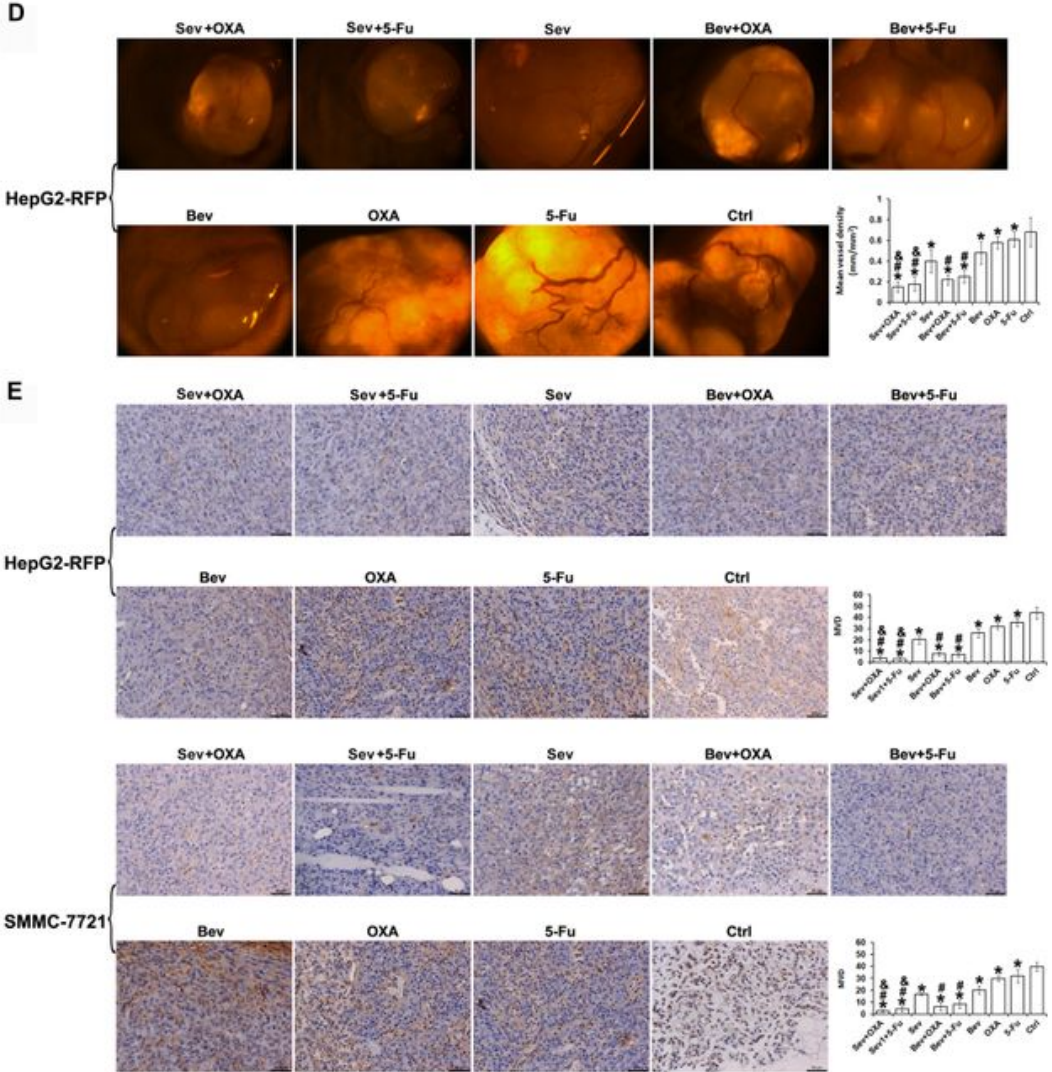

\section{Figure 4}

Anti-angiogenic effects of Sev combined with chemotherapy in zebrafish, chicken embryos, and mouse HCC xenografts. (A) Zebrafish embryonic-angiogenic assessment. After $48 \mathrm{~h}$ of drug administration in zebrafish embryos, the inhibition rate of ISV formation in Sev combined with 5-Fu or OXA groups was significantly enhanced $(* P<0.05$, compared with the control group; $\# P<0.05$, compared with the corresponding monotherapy groups; \& $\mathrm{P}<0.05$, compared with the Bev + chemotherapy groups). (B) The 
RT-qPCR testing of zebrafish embryonic tissue showed that the downregulation of VEGF mRNA level in the Sev + chemotherapy groups was markedly lower. 1: Sev+OXA, 2: Sev+5-Fu, 3: Sev, 4: Bev+OXA, 5: Bev+5-Fu, 6: Bev, 7: OXA, 8: 5-Fu, 9: control group. After $72 \mathrm{~h}$ of drug administration, the anti-angiogenic effects in CAM were significantly stronger in the Sev + chemotherapy groups ( ${ }^{*} \mathrm{P}<0.05$, compared with the control group; $\# \mathrm{P}<0.05$, compared with the corresponding monotherapy groups; \& $P<0.05$, compared with the Bev + chemotherapy groups). (D) Sev in combination with chemotherapy suppressed the angiogenesis in HCC xenograft tumor. Mice-bearing HepG2-RFP cell xenografts were administered with different doses of Sev or Bev in combination with 5-Fu or OXA. The surface of the tumor blood vessel density was calculated using Image-Pro software ( ${ }^{*} \mathrm{P}<0.05$, compared with the control group; \# $\mathrm{P}$ $<0.05$, compared with the corresponding monotherapy groups; \& $\mathrm{P}<0.05$, compared with the Bev + chemotherapy groups). (E) Nude mice inoculated with HepG2-RFP cell xenografts were given the indicated drugs for 21 days. At day 22, CD31 was assessed by immunohistochemical analysis $\left({ }^{\star} P<0.05\right.$, compared with the control group; \# $\mathrm{P}<0.05$, compared with the corresponding monotherapy groups; \& $\mathrm{P}$ $<0.05$, compared with the Bev + chemotherapy groups). 
A
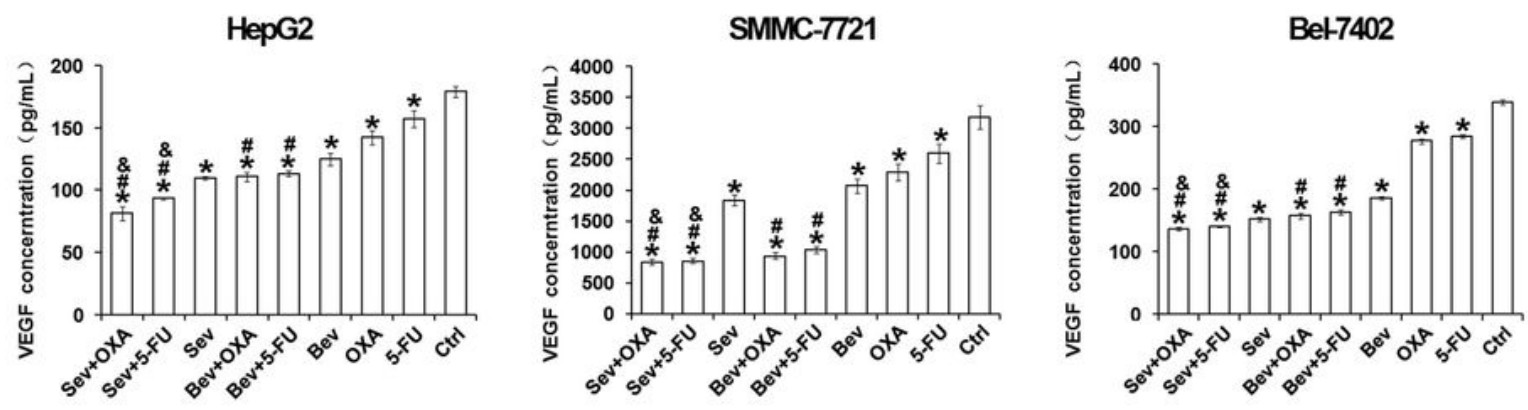

HUVEC
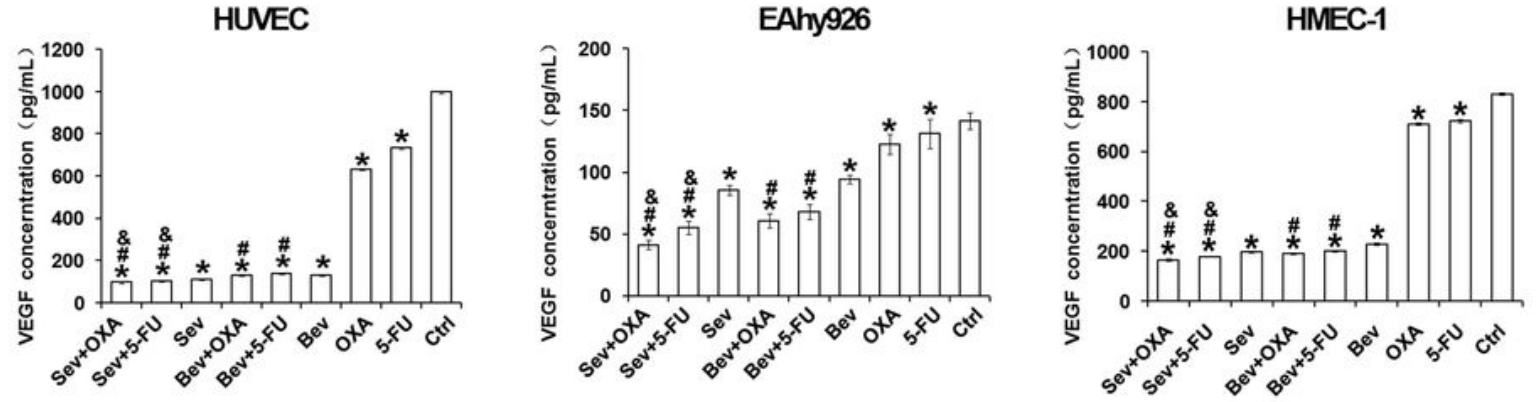

B

HepG2

SMMC-7721

Bel-7402
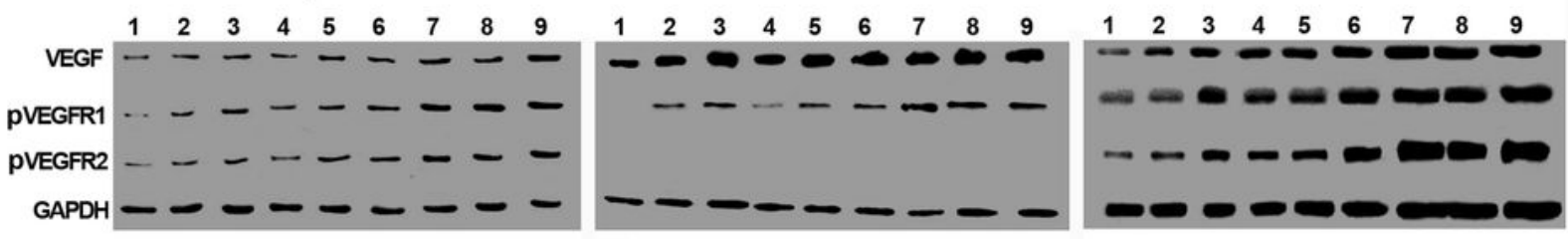

HUVEC

EAhy926

HMEC-1
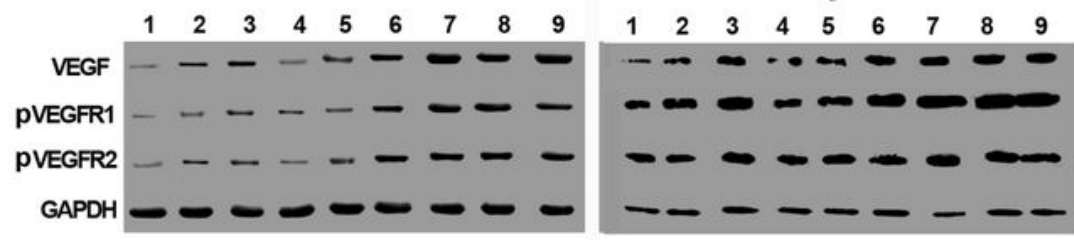

C

HepG2-RFP

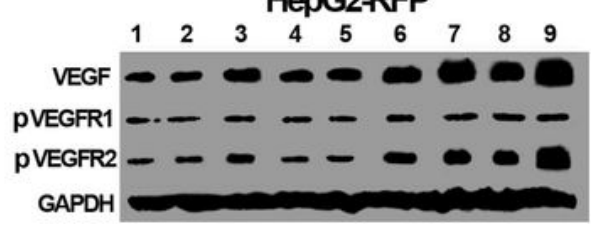

Figure 5

Sev in combination with chemotherapy inhibited the VEGF/VEGFR pathway in HCC cells. The HCC cell lines (HepG2, SMMC-7721, and Bel7402) and endothelial cells (HUVEC, EAhy926, and HMEC-1) were treated with the different concentrations of Sev or Bev in combination with 5-Fu or OXA for $48 \mathrm{~h}$. (A) ELISA was applied to detect the VEGF level in the cell culture medium ( ${ }^{*} P<0.05$, compared with the control group; \# $\mathrm{P}<0.05$, compared with the corresponding monotherapy groups; \& $\mathrm{P}<0.05$, compared 
with the Bev + chemotherapy groups). (B) Total proteins were extracted for immunoblotting of VEGF, pVEGFR1, pVEGFR2, with GAPDH as the loading control. (C) Nude mice-bearing HepG2-RFP cell xenografts were administered with the drug for 21 days. At day 22, mice were sacrificed and tumor tissues were processed for immunoblotting of VEGF, pVEGFR1, and pVEGFR2, with GAPDH as the loading control. 